\title{
Proximal tibial fractures: early experience using polyaxial locking-plate technology
}

\author{
Vassilios S. Nikolaou • Hiang Boon Tan • \\ George Haidukewych • Nikolaos Kanakaris • \\ Peter V. Giannoudis
}

Received: 18 June 2010 /Revised: 25 October 2010 /Accepted: 26 October 2010 /Published online: 25 November 2010

(C) Springer-Verlag 2010

\begin{abstract}
Between 2004 and 2009, 60 patients with proximal tibial fractures were included in this prospective study. All fractures were treated with the polyaxial lockedplate fixation system (DePuy, Warsaw, IN, USA). Clinical and radiographic data, including fracture pattern, changes in alignment, local and systemic complications, hardware failure and fracture union were analysed. The mean follow-up was 14 (12-36) months. According to the Orthopaedic Trauma Association (OTA) classification,
\end{abstract}

Work attributed to Academic Unit, Trauma and Orthopaedic Surgery, Clarendon Wing, Leeds General Infirmary, Great George Street, Leeds, LS1 3EX, UK

Orthopaedic Trauma Service, Florida Orthopaedic Institute, 13020 Telecom Parkway, Temple Terrace, FL 33637.

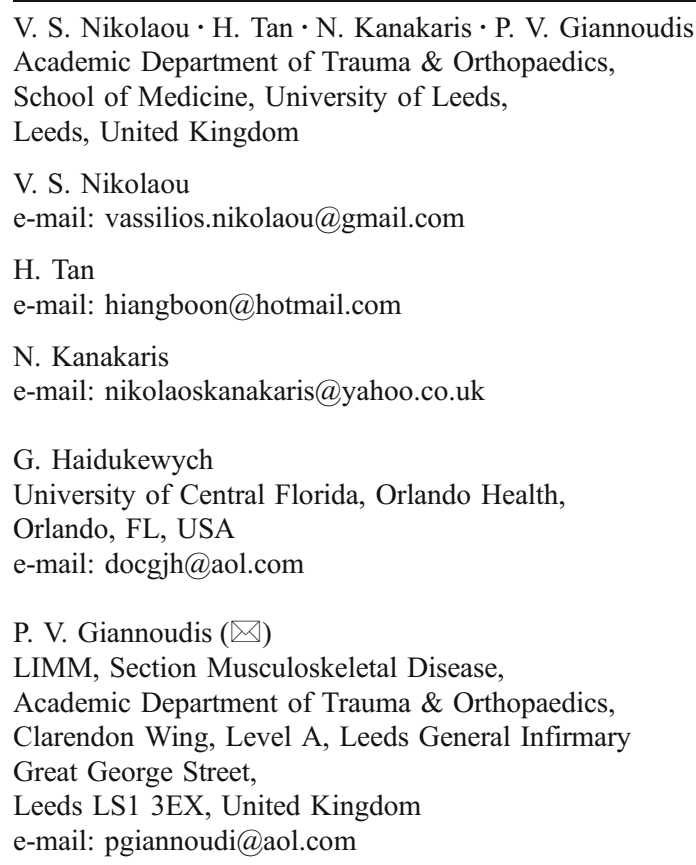

e-mail: pgiannoudi@aol.com

there were five 41-A, 28 41-B and 27 41-C fractures. Fractures were treated percutaneously in $30 \%$ of cases. Double-plating was used in 11 cases. All but three fractures progressed to union at a mean of $3.2(2.5-5)$ months. There was no evidence of varus collapse as a result of polyaxial screw failure. No plate fractured, and no screw cut out was noted. There was one case of lateral joint collapse $\left(>10^{\circ}\right)$ in a patient with open bicondylar plateau fracture. The mean Knee Society Score at the time of final follow-up was 91 points, and the mean functional score was 89 points. The polyaxial locking-plate system provided stable fixation of extra-articular and intra-articular proximal tibial fractures and good functional outcomes with a low complication rate.

\section{Introduction}

Various treatment modalities can be used to treat proximal tibial fractures. Nonoperative treatment with traction, casts or braces has been reported to produce good results in selected cases, but complications such as loss of reduction, prolonged hospital stay and poor functional outcome can be expected. Consequently, the nonoperative treatment option is limited to minimally or undisplaced fractures without major soft-tissue or neurovascular injury that are generally considered stable [1]. Arthroscopically assisted fixation and minimal percutaneous pinning have also been reported to give good results, but these modalities are suitable for simple split-depression and local compression fractures [2]. For decades, open reduction and internal fixation with plates has been the treatment of choice, especially for fractures with intraarticular extension. However, this treatment modality often requires invasive exposure of the fracture zone, which may endanger surrounding soft tissues, thus compromising the biological environment for fracture healing. External fixation 
with a circular or hybrid external fixator is another effective treatment option, but the problems of nonunion, pin-track infection, septic arthritis, pain, discomfort and joint stiffness cannot be underestimated [3].

The introduction of the locking-plate technology has offered another alternative to treat proximal tibia fractures. Locked plates, or internal fixators, are designed to achieve an angular-stable connection between the screw head and the force carrier without the necessity of frictional forces between the implant and the bone [4]. Moreover, the technology of minimally invasive percutaneous osteosynthesis (MIPO) can be easily applied. This allows minimum soft-tissue damage at the fracture site, offering all the theoretical advantages of biological osteosynthesis [5]. Recently, locking plates have been designed to have polyaxial technology, thus allowing screw positioning with a variable degree of freedom for fixation. This versatility can be considered as an advantage, allowing for more stable osteosynthesis of highly comminuted or osteoporotic fractures. Even though the polyaxial technology has been used for many years for fixation systems in spinal surgery, its application in plate osteosynthesis is still limited.

In our institutions, we have been using a tibial lockingplate system characterised by polyaxial technology (DePuy, Warsaw, IN, USA). The literature regarding its efficacy for stabilisation of proximal tibial fractures is sparse. The purpose of this study, therefore, was to evaluate a series of proximal tibial fractures treated with a polyaxial locking-plate system in order to document the performance of this new device and to review the clinical and radiological results.

\section{Patients and methods}

Between January 2004 and September 2009, all patients treated in our institutions (two level 1 trauma centres in the USA and UK) with polyaxial POLYAX plate (DePuy) technology for proximal tibial fractures were eligible to participate. Exclusion criteria were cases that had been treated by other means of osteosynthesis and patients who were lost to follow-up.

A series of patients included in this report ( 25 cases) has been described in an earlier study that presented mixed results of the polyaxial locked-plate fixation in periarticular fractures of the knee [6]. Results of this previous work showed that these variable-axis locking plates performed well, with a high rate of fracture union and no evidence of varus collapse due to failure of the polyaxial screw fixation in a combined series of distal femur and proximal tibial fractures. We wanted to further investigate the feasibility of the POLYAX plating technology in a larger series of patients, particularly in relation to proximal tibial fractures. For this reason, we combined the cases treated between the two institutions, as previously described.
All the hospital charts, records and radiographs were retrieved and retrospectively analysed. All patients were treated by two experienced surgeons who were familiar with the principles of the MIPO technique. All fractures were classified according to the Orthopaedic Trauma Association (OTA) system. Patient demographics, cause and pattern of injury, clinical and radiographic data including fracture pattern, changes in alignment, local and systemic complications, hardware failure and time to union were documented and analysed. Preoperatively, besides radiography, computed tomography (CT) scanning was obtained for type B and C fractures. The timing of surgery depended on the soft-tissue conditions. Surgery was delayed if the fractures had established severe soft-tissue swelling and skin blistering. In these cases, a temporary spanning external fixation was used for soft-tissue resuscitation prior to definitive fracture reconstruction.

Surgery was performed under general anaesthesia on a standard radiolucent table. Where possible, MIPO was performed. Double plating (medial and lateral) was used in fractures with a displaced coronal split component of the posterior aspect of the medial tibial plateau. This was the only indication for the use of double plates. Open fractures were treated by emergency débridement, tetanus prophylaxis and intravenous antibiotics. Where necessary, bone autograft or allograft was used for tibial metaphyseal defects. Postoperatively, all patients were given intravenous prophylactic antibiotics and mechanical or/and pharmacological prophylaxis against deep venous thrombosis. Rehabilitation was started on the second postoperative day with static quadriceps excercises and continuous passive motion of the knee joints. Approximately four to six weeks after surgery, partial weight bearing was encouraged. Full weight bearing was not permitted until consolidation of the fracture site. Follow-up routine anteroposterior, lateral, and oblique radiographs were obtained at two, four, eight and then every four weeks until clinical and radiological fracture union had been established. Radiological union was defined as the presence of trabeculation crossing the fracture on radiographs of at least three cortices. Clinical union was defined as the presence of painless full weight bearing. To assess malalignment, radiographs at the latest follow-up were compared with the first postoperative ones. Tibial alignment in both planes was measured by comparing lines drawn along the tibial shaft and parallel to the articular surface. Malalignment was defined as the presence on radiographs of more than $5^{\circ}$ angulation in any plane at final last follow-up. Other radiographic indications of loss of reduction, such as a step at the articular surface $>2 \mathrm{~mm}$, were also recorded and documented. Functional assessment was performed using the Knee Society criteria and reported as knee scores and function scores. The mean follow-up was 14 (12-36) months. 


\section{Results}

In total, 60 patients met the inclusion criteria, with a mean age of 52.8 (range 18-86) years. According to the OTA classification, there were five 41-A, 28 41-B and 27 41-C fractures. Seven were open fractures. The majority of patients $(54 / 60)$ presented with isolated injuries. The mechanism of injury included falls from a height (50\%), motor-vehicle accidents (45\%) and other causes (assaults) in $5 \%$ of cases. Twenty-eight patients required temporary external fixation before the final surgery. From the 60 patients, five were lost to follow-up and therefore 55 patients formed the report of this study. Preoperative patient details are summarised in Table 1.

At surgery, 27 patients required bone grafting in the form of croutons, or iliac crest autograft. In eight of them, allograft in combination with growth factors was used. Double plating was performed in 11 patients according to the previously mentioned indications. Overall, the MIPO technique was used in 18 patients, (Table 2).

All fractures but three progressed to union (94.5\%). Delayed union was observed in one patient who was a heavy smoker. His fracture healed following treatment with the ultrasound bone healing system (Exogen ${ }^{\circledR}$, Smith \& Nephew, Memphis, TN, USA) six months following surgery. All other fractures were healed at a mean of 3.2 (range 2.5-5) months. Two fractures that failed to unite were complicated by deep sepsis and required further intervention. Both these patients had bicondylar (OTA type $41 \mathrm{C} 3$ ) fracture of the tibial plateau. One of them was an insulin-dependent diabetic with severe peripheral neuropathy. He had an early infection and unfortunately required above the knee amputation. The other fracture that failed to unite was that of a 70-year-old woman with an OTA 41B3

Table 1 Preoperative patient details

\begin{tabular}{ll}
\hline Details & Number (\%) \\
\hline Patients number & 60 \\
Mean age (years) & $52.8(18-86)$ \\
Male/female ratio & $29 / 31$ \\
Right/left ratio & $33 / 27$ \\
Isolated fracture & 54 \\
Mechanism of injury (\%) & Fall from a height (50\%), RTA (45\%) \\
& Other causes 5\% \\
Fracture type (OTA) & $41-\mathrm{A}: 5$ \\
& $41-\mathrm{B}: 28$ \\
& $41-\mathrm{C}: 27$ \\
Initial external fixation & Yes: 28 \\
Open/closed & No: 32 \\
\hline
\end{tabular}

RTA road traffic accident, OTA Orthopaedic Trauma Association
Table 2 Surgical details

\begin{tabular}{ll}
\hline & Number \\
\hline Average time to surgery (days) & $4(1-9)$ \\
Surgery within $24 \mathrm{~h}$ & 14 \\
External fixator used for reduction (intraoperatively) & 17 \\
Mean operation time (min) (skin to skin) & 68 (52-95) \\
Primary bone grafting & Yes: 27 \\
& No: 33 \\
Arthroscopic assistance & None \\
Double plating & Yes: 11 \\
& No: 49 \\
MIPO technique & Yes: 18 \\
& No: 42 \\
\hline
\end{tabular}

MPO minimal invasive plate osteosynthesis

fracture. She was treated with a lateral POLYAX plate with no intraoperative complications. Her fracture failed to unite and collapsed. The plate was removed after nine months, and she underwent a total knee replacement (TKR) six months later (Fig. 1). The third patient had a Gustilo and Anderson [7] grade 3-A open fracture, and when a deep infection developed, was treated with wound débridement, removal of hardware and eventually osteoarticular allograft reconstruction (Fig. 2).

One patient required fasciotomies for compartment syndrome, but the fracture finally united uneventfully. One patient had peroneal nerve palsy with foot drop that recovered, and his fracture healed uneventfully with good final outcome. Superficial infection was treated successfully with a short course of antibiotics in two cases. No other complications were recorded. No plate fractured or screw cut-out occurred. Radiographic evaluation at the time of the latest follow-up revealed two cases of lateral joint collapse $\left(>10^{\circ}\right)$. The first patient had an open bicondylar plateau fracture and developed deep sepsis and nonunion. The second patient went to nonunion, fracture collapse and eventually plate removal and TKR. There was no evidence of varus collapse as a result of polyaxial screw failure in any patient. The mean Knee Society Score at the time of the latest follow-up was 91 (59-100) points, and the mean functional score was 89 (54-100) points. Postoperative complications and outcome are summarised in Table 3.

\section{Discussion}

For many years, treating proximal tibial fractures has been the subject of much controversy regarding both the indications for surgical intervention and the specific type of intervention to be employed. These fractures often affect patients during the most productive years of their lives, 

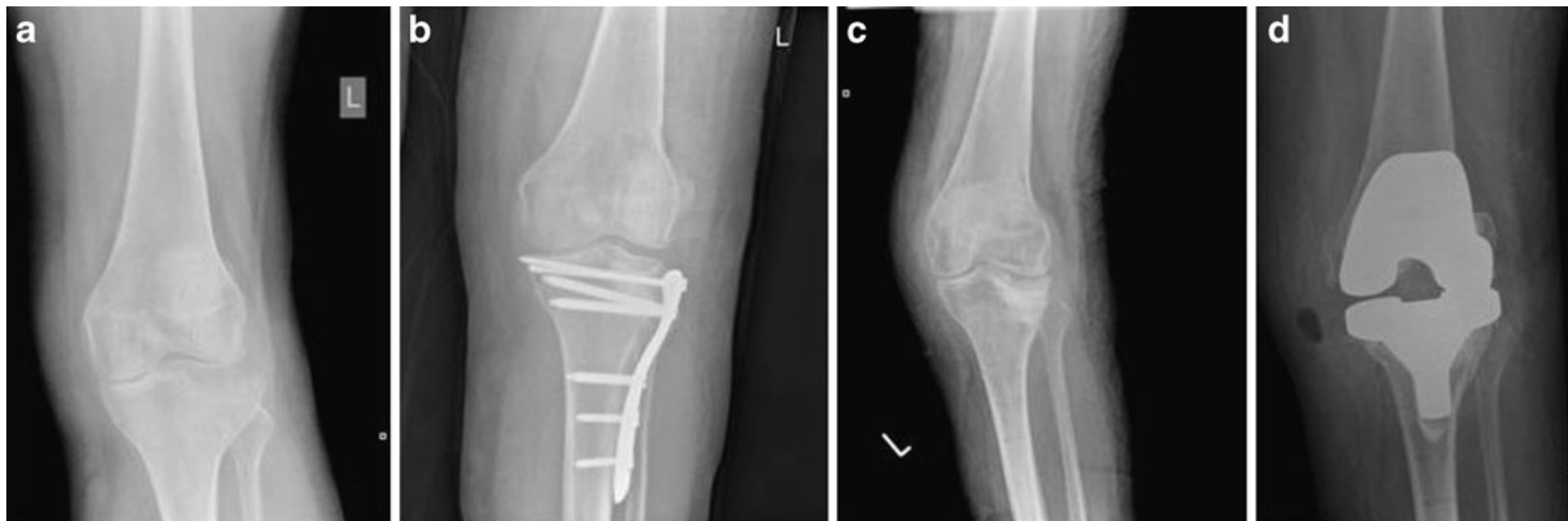

Fig. 1 A 70-year-old woman with an Orthopaedic Trauma Association (OTA) 41B-3 fracture. a Preoperative X-ray. b One month postoperatively. $\mathbf{c}$ Nine months later, plate was removed as her fracture

with potentially devastating consequences. Especially in intra-articular fractures, inadequate treatment may result in joint instability and deformity coupled with a restricted range of motion $[8,9]$. Open reduction and rigid internal fixation, according to the principles of Association for Osteosynthesis/Association for the Study of Internal Fixation (AO/ASIF), has been the treatment of choice for decades. This treatment modality has yielded satisfactory short- and long-term results in many series. However, especially in the setting of high-energy, complex tibialplateau fractures, conventional plating has been associated with a high rate of wound complications and deep sepsis [10]. In these cases, excessive dissection through the injured soft-tissue envelope and devitalisation of bone fragments are known factors contributing to the development of such complications. Additionally, conventionalplate osteosynthesis requires compression of the plate to the bone and relies on friction at the bone-plate interface. This is inevitably associated with biological pitfalls associated with compression of periosteal blood supply and compromise of the vascularity of the fracture. As a result, failed to unite and collapsed. $\mathbf{d}$ She underwent total knee replacement (TKR) 6 months later

complications such as infection, hardware failure, delayed union and nonunion are more likely to occur.

Recently, locking plates, or internal fixators, have been designed to allow for less plate to bone contact without compromising stability. The screw holes are modified to allow the screw to "lock" into the plate, thus converting a plate/screw construction into a fixed-angle device with multiple points of fixation [11]. This design allows for minimal vascular damage to the periosteum. Moreover, locking plates can be particularly effective in treating osteoporotic bones [12]. The evolution of locking-plate technology has led to newly designed, anatomically preshaped plates for different fractures, such as proximal humerus, proximal and distal femur and proximal and distal tibia [13]. These plates are also designed to enhance the surgeon's ability to pass the plate in a submuscular or subcutaneous manner for minimally invasive application [13].

Previous series of proximal tibia fractures treated with locking plates have shown good results with low complication rates [14]. Table 4 summarises some of the published
Fig. 2 A 31-year-old man with Orthopaedic Trauma Association (OTA) $41 \mathrm{C} 3$ fracture. a Preoperative X-ray. b)1 week post-operatively. c 2.5 years postoperatively
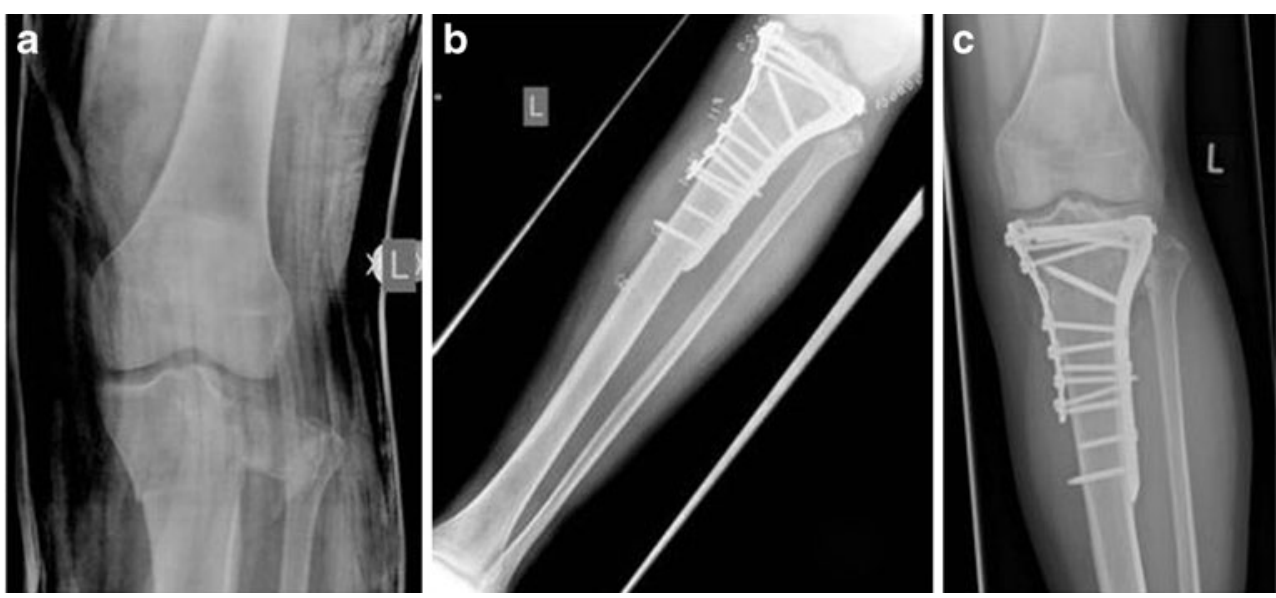
Table 3 Postoperative complications and outcome

Patient characteristics

Patients with follow-up ( $n$ )

55

Mean follow-up time (range)

14 months (12-36)

Union rate (\%)

94.5

Radiographic healing time (months)

3.2 (range $2.5-5$ )

Complications (12)

Seroma

0

Hematoma

0

Nerve palsy

Superficial infection

Deep infection

Deep venous thrombosis

Hardware failure

Hardware irritation

Nonunion

Fasciotomies

Loss of reduction

Loss of alignment

Implants removal

Revision open reduction internal fixation

Knee Society Score

Mean knee score (range)

$91(59-100)$

Mean functional score (range)

$89(54-100)$

series of patients with proximal tibia fracture treated with the locking-plate technology as well as the reported major complications. Union rate ranged between $80 \%$ and $100 \%$ (mean 93.4\%). In contrast to those results, Phisitkul et al. [15] reported a higher rate of complications and pitfalls. In a retrospective review of 43 adult patients with complex fractures of the proximal tibia (35 were intra-articular and 18 of those were comminuted C3 fractures) treated with a fixed-angle locking plate [less invasive stabilisation system (LISS)] placed on the lateral side of the proximal tibia, the authors reported that only $32 \%$ of the fractures healed without complications. Gosling et al. [16], in a multicentre study, prospectively followed a case series of 68 patients with $69 \mathrm{AO} / \mathrm{ASIF}$ 41-C-type fractures treated with the LISS for the proximal lateral tibia. Sixty-two of these patients were available for final follow-up: $25 \%$ had postoperative radiographs that revealed malreduction, $14 \%$ of fractures had secondary loss of reduction and 5\% did not achieve union. More recently, Jiang et al. [17], in a prospective study, compared the clinical outcomes of conventional double plating and a single lateral LISS for the repair of bicondylar tibial plateau fractures. They found significantly higher incidence of postoperative malalignment of the proximal tibia, and a trend towards significance of a higher incidence of symptomatic hardware irritation in the
LISS group compared with the double-conventional-plate group.

Phisitkul et al. [15] recognised the inaccurate fracture reduction before plate application as a aggravating factor for the high percentage of postoperative malalignment. Additionally, they emphasised the disadvantage of the LISS system regarding lack of screw-length options and concluded that the fixed angle of the screw direction limits the capability of enhancing fixation to the medial cortex or medial subchondral bone without the risk of joint penetration. Gosling et al. [16] additionally stressed the importance of the surgeon's experience in the locking-plate technique, especially when combined with the MIPO technique.

In this study, the POLYAX proximal tibial plate, made of TiMAX titanium alloy (DePuy) was used in all patients. In this implant, locking screws can be positioned at customised angles best suited to each patient's anatomy. This is the main advantage of this new implant design. As a result, screws can be angled cephalad or caudal to achieve optimal positioning in the tibial metaphysis in areas with good bone stock. Additionally, the danger of penetrating into the knee joint is minimal. However, a polyaxial screw has less resistance to bending forces than a fixed locking screw. This is because polyaxial plates rely on an additional interface between the plate and screw head in a way that overall stability depends on friction from hoop stresses rather than from threads. More specifically, the new polyaxial plate uses a threaded expanding bushing contained in the head of the plate. The bushing can angulate within the plate, allowing screw positioning in different angles. When the screw head is fully seated in the bushing, it exerts a hoop stress that causes friction between the bushing and the plate. Results of this study showed that this friction, even though biomechanically inferior to the fixed locking screw, was adequate to maintain stable osteosynthesis.

The vast majority of fractures $(94.5 \%)$ in this patient series progressed to union. These results are in accordance with previously published reports using fixed-angle locking devices [18]. Moreover, complications occurred in only three patients $(5.5 \%)$. The very low complications rate, especially in terms of infection, malunion and nonunion can be attributed to the effectiveness of the implant used and to the fact that very experienced surgeons performed the procedures. From the $2741-\mathrm{C}$ fractures, only 11 were treated with the double-plating technique. Previous studies have shown that treatment of bicondylar proximal tibial fractures with one single lateral locking plate can result in postoperative loss of reduction in a high percentage of patients. The traditional lateral-plating systems offers little resistance to varus deformity, thus adjuvant medial neutralisation plating has been advocated to increase stability in fracture patterns with metaphyseal comminution. Gosling et 
Table 4 Published series of proximal tibia fractures treated with locking plates

\begin{tabular}{|c|c|c|c|c|c|c|c|}
\hline Publication & Year & Study design & Fracture site & $\begin{array}{l}\text { No. of } \\
\text { fractures with } \\
\text { follow-up }\end{array}$ & $\begin{array}{l}\text { Locking } \\
\text { plate } \\
\text { used }\end{array}$ & $\begin{array}{l}\text { Overall } \\
\text { complications } \\
\text { (major/ minor) }\end{array}$ & $\begin{array}{l}\text { Percent } \\
\text { successful } \\
\text { outcome } \\
\text { (union) }\end{array}$ \\
\hline Cole et al & 2003 & $\begin{array}{l}\text { Prospective } \\
\text { clinical trial }\end{array}$ & Bicondylar tibia plateau & 54 & LISS * & $8 / 9$ & $96 \%$ \\
\hline Schütz et al & 2003 & $\begin{array}{l}\text { Prospective } \\
\text { study }\end{array}$ & $\mathrm{AO} 41-\mathrm{C}, \mathrm{AO} 41-\mathrm{A} 7, \mathrm{AO} 42$ & 20 & LISS & $3 / 3$ & $80 \%$ \\
\hline Ricci et al & 2004 & $\begin{array}{l}\text { Prospective } \\
\text { clinical trial }\end{array}$ & $\begin{array}{l}\text { Comminuted proximal tibia } \\
\text { metaphyseal fractures ( } 41 \mathrm{~A} 3 \text {, } \\
41 \mathrm{C} 2 \text {, or } 41 \mathrm{C} 3)\end{array}$ & 38 & LISS & $5 / 5$ & $97 \%$ \\
\hline Boldin et al & 2006 & $\begin{array}{l}\text { Prospective } \\
\text { study }\end{array}$ & $\mathrm{AO} 41-\mathrm{A}, \mathrm{AO} 41-\mathrm{C}$ & 25 & LISS & $5 / \mathrm{NA}$ & $96 \%$ \\
\hline Stannard et al & 2004 & $\begin{array}{l}\text { Prospective } \\
\text { study }\end{array}$ & OTA 41-C & 34 & LISS & $5 / 8$ & $100 \%$ \\
\hline Cole et al & 2004 & $\begin{array}{l}\text { Prospective } \\
\text { study }\end{array}$ & OTA 41 and OTA 42 & 77 & LISS & $7 / 8$ & $91 \%$ \\
\hline Egol et al & 2004 & $\begin{array}{l}\text { Retrospective } \\
\text { study }\end{array}$ & OTA 41-C & 36 & LISS & $1 / 5$ & $94 \%$ \\
\hline
\end{tabular}

OTA Orthopaedic Trauma Association, LISS Less Invasive Skeletal System , NA not available

al. [16], in a multicentre study, reported $23 \%$ postoperative malalignment and $14 \%$ loss of alignment when high-energy bicondylar proximal tibia fractures where treated with laterally placed LISS plate only. Phisitkul et al. [15] reported immediate postoperative and delayed loss of alignment in $22 \%$ and $8 \%$ of cases, respectively, when lateral LISS plate was used in similar fractures. Egol et al. [19] published a laboratory study comparing dual plating with a single lateral LISS plate. After cyclical loading, they showed a significant difference in inferior displacement of the medial fragment, with an average of $9.6 \mathrm{~mm}$ for the LISS group compared with $4.9 \mathrm{~mm}$ for the dual-plating group $(p=0.05)$. Similar results yielded by Ratcliff et al. [20] with their cadaveric biomechanical study show that in the setting of a vertically oriented fracture in a medial tibial plateau without comminution, the medial buttress plate provides significantly greater stability in static loading and improved stability with cyclic loading in comparison with a single lateral locking-plate stabilisation . In our series, only two patients had lateral collapse $>10^{\circ}$. The first patient was treated with double plating and developed deep sepsis and nonunion. The second patient's fracture failed to unite, and a secondary collapse of the fracture occurred. There was no evidence of varus collapse as a result of polyaxial screw failure in any patient. These positive results are indicative of the biomechanical advantages that the new polyaxial implant can offer.

We used primary bone grafting in $45 \%$ of patients. In other series, this percentage ranges between $57 \%$ and $100 \%$ $[21,22]$. The mean Knee Society Score and functional score in this study were 91 and 89 points, respectively. These numbers include all patients, irrespective of the fracture pattern and severity of the injury and are comparable with previously published series using locking [23] or conventional plates [24].

We are aware of the limitations of this study. Patients were treated in two different trauma centres. Additionally, patients with multiple fracture patterns, open and closed fractures and single lateral- and bilateral-plate osteosynthesis were included. Inevitably, this makes it difficult to draw definitive conclusions. The relative small number of patients is another drawback. Additionally, there are studies that support the view that the incidence of post-traumatic degenerative osteoarthritis following intra-articular fractures of the knee tends to develop from six to eight years after injury [25]. Consequently, the follow-up period of this study is short, especially for the identification of such longterm complications. However, the objective was to evaluate the efficacy of this new device.

In conclusion, the POLYAX plate system provided stable fixation in this series of patients with proximal tibial fractures. The overall complication rate was low, and the functional outcome satisfactory in the mean follow-up time of 14 months. We found $94.5 \%$ of simple and complex fractures progressed to union, and the deep infection rate was $3.3 \%$. However, the fixation technique is demanding, and the surgeon needs considerable experience with locking-plate techniques and especially in percutaneous techniques. Further prospective, randomised studies are desirable to confirm our findings and to eventually draw safer conclusions.

Acknowledgement The authors express their gratitude to all staff of the two institutions for their support in this study. 


\section{References}

1. Bono CM, Levine RG, Rao JP, Behrens FF (2001) "Nonarticular proximal tibia fractures: treatment options and decision making". J Am Acad Orthop Surg 9:176-186

2. Pogliacomi F, Verdano MA, Frattini M, Costantino C, Vaienti E, Soncini G (2005) "Combined arthroscopic and radioscopic management of tibial plateau fractures: report of 18 clinical cases". Acta Biomed 76:107-114

3. Mallik AR, Covall DJ, Whitelaw GP (1992) "Internal versus external fixation of bicondylar tibial plateau fractures". Orthop Rev 21:1433-1436

4. Schutz M, Sudkamp NP (2003) "Revolution in plate osteosynthesis: new internal fixator systems". J Orthop Sci 8:252-258

5. Baumgaertel F, Buhl M, Rahn BA (1998) "Fracture healing in biological plate osteosynthesis". Injury 29(Suppl 3):C3-C6

6. Haidukewych G, Sems SA, Huebner D, Horwitz D, Levy B (2007) "Results of polyaxial locked-plate fixation of periarticular fractures of the knee". J Bone Joint Surg Am 89:614-620

7. Gustilo RB, Anderson JT (1976) "Prevention of infection in the treatment of one thousand and twenty-five open fractures of long bones: retrospective and prospective analyses". J Bone Joint Surg Am 58:453-458

8. Yu B, Han K, Ma H, Zhang C, Su J, Zhao J, Li J, Bai Y, Tang H (2009) "Treatment of tibial plateau fractures with high strength injectable calcium sulphate". Int Orthop 33:1127-1133

9. Stevens DG, Beharry R, McKee MD, Waddell JP, Schemitsch EH (2001) "The long-term functional outcome of operatively treated tibial plateau fractures". J Orthop Trauma 15:312-320

10. P. F. Lachiewicz and T. Funcik, (1990) "Factors influencing the results of open reduction and internal fixation of tibial plateau fractures" Clin Orthop Relat Res: 210-215.

11. Greiwe RM, Archdeacon MT (2007) "Locking plate technology: current concepts". J Knee Surg 20:50-55

12. Fulkerson E, Egol KA, Kubiak EN, Liporace F, Kummer FJ, Koval KJ (2006) "Fixation of diaphyseal fractures with a segmental defect: a biomechanical comparison of locked and conventional plating techniques". J Trauma 60:830-835

13. Smith WR, Ziran BH, Anglen JO, Stahel PF (2007) "Locking plates: tips and tricks". J Bone Joint Surg Am 89:2298-2307
14. Singh S, Patel PR, Joshi AK, Naik RN, Nagaraj C, Kumar S (2009) "Biological approach to treatment of intra-articular proximal tibial fractures with double osteosynthesis". Int Orthop 33:271-274

15. Phisitkul P, McKinley TO, Nepola JV, Marsh JL (2007) "Complications of locking plate fixation in complex proximal tibia injuries". J Orthop Trauma 21:83-91

16. Gosling T, Schandelmaier P, Muller M, Hankemeier S, Wagner M, Krettek C (2005) "Single lateral locked screw plating of bicondylar tibial plateau fractures". Clin Orthop Relat Res 439:207-214

17. Jiang R, Luo CF, Wang MC, Yang TY, Zeng BF (2008) "A comparative study of Less Invasive Stabilization System (LISS) fixation and two-incision double plating for the treatment of bicondylar tibial plateau fractures". Knee 15:139-143

18. Stannard JP, Wilson TC, Volgas DA, Alonso JE (2004) "The less invasive stabilization system in the treatment of complex fractures of the tibial plateau: short-term results". J Orthop Trauma 18:552-558

19. Egol KA, Su E, Tejwani NC, Sims SH, Kummer FJ, Koval KJ (2004) "Treatment of complex tibial plateau fractures using the less invasive stabilization system plate: clinical experience and a laboratory comparison with double plating". J Trauma 57:340-346

20. Ratcliff JR, Werner FW, Green JK, Harley BJ (2007) "Medial buttress versus lateral locked plating in a cadaver medial tibial plateau fracture model". J Orthop Trauma 21:444-448

21. Bansal MR, Bhagat SB, Shukla DD (2009) "Bovine cancellous xenograft in the treatment of tibial plateau fractures in elderly patients". Int Orthop 33:779-784

22. Muggler E, Huber D, Burri C (1975) "[Results of surgical treatment of 225 tibial-head fractures]". Chirurg 46:348-352

23. Boldin C, Fankhauser F, Hofer HP, Szyszkowitz R (2006) "Threeyear results of proximal tibia fractures treated with the LISS". Clin Orthop Relat Res 445:222-229

24. Benirschke SK, Agnew SG, Mayo KA, Santoro VM, Henley MB (1992) "Immediate internal fixation of open, complex tibial plateau fractures: treatment by a standard protocol". J Orthop Trauma 6:78-86

25. Manidakis N, Dosani A, Dimitriou R, Stengel D, Matthews S, Giannoudis P (2010) "Tibial plateau fractures: functional outcome and incidence of osteoarthritis in 125 cases". Int Orthop 34:565570 